\title{
Ternary Free-Energy Entropic Lattice Boltzmann Model with a High Density Ratio
}

\author{
M. Wöhrwag, ${ }^{1,2}$ C. Semprebon, ${ }^{3}$ A. Mazloomi Moqaddam, ${ }^{2,4}$ I. Karlin, ${ }^{2, *}$ and H. Kusumaatmaja ${ }^{1, \dagger}$ \\ ${ }^{1}$ Department of Physics, Durham University, South Road, Durham DH1 3LE, United Kingdom \\ ${ }^{2}$ Department of Mechanical and Process Engineering, ETH Zurich, Zurich CH-8092, Switzerland \\ ${ }^{3}$ Smart Materials \& Surfaces Laboratory, Northumbria University, Newcastle upon Tyne NE1 8ST, United Kingdom \\ ${ }^{4}$ Laboratory for Multiscale Studies in Building Physics Empa, Swiss Federal Laboratories for Materials Science and Technology, \\ 8600 Dübendorf, Switzerland
}

(Received 14 October 2017; published 8 June 2018)

\begin{abstract}
A thermodynamically consistent free energy model for fluid flows comprised of one gas and two liquid components is presented and implemented using the entropic lattice Boltzmann scheme. The model allows a high density ratio, up to the order of $O\left(10^{3}\right)$, between the liquid and gas phases, and a broad range of surface tension ratios, covering partial wetting states where Neumann triangles are formed, and full wetting states where complete encapsulation of one of the fluid components is observed. We further demonstrate that we can capture the bouncing, adhesive, and insertive regimes for the binary collisions between immiscible droplets suspended in air. Our approach opens up a vast range of multiphase flow applications involving one gas and several liquid components.
\end{abstract}

DOI: 10.1103/PhysRevLett.120.234501

Multiphase flows comprised of one gas and several liquid components are of considerable scientific interest due to their broad range of applications. The collision between oil and water droplets is a key ignition step in combustion engines, where the collision parameters can be varied to control the effective burning rate [1]. The presence of an immiscible crude oil layer on the sea surface alters the processes occurring during raindrop impact, with consequences for marine aerosol creation and oil spill dispersal [2]. In advanced oil recovery, considerable gain can be achieved by alternately displacing the oil by air and water in the so-called immiscible water-alternating-gas displacement process [3]. Infusing porous materials with lubricants results in composite surfaces, known as lubricant impregnated surfaces [4-7], with superior nonwetting and dragreduction properties.

Despite the wide-ranging applications, suitable quantitative models for studying these phenomena are surprisingly still lacking. Most simulations to date have focussed on either single-component liquid-gas systems with a high density ratio [8-12] or multicomponent flows with an equal (or similar) density ratio [13-19]. In contrast, our aim here is to demonstrate an accurate and flexible model that can predict complex interfacial dynamics of ternary systems with a significant ratio

Published by the American Physical Society under the terms of the Creative Commons Attribution 4.0 International license. Further distribution of this work must maintain attribution to the author(s) and the published article's title, journal citation, and DOI. between the liquid and gas densities, up to the order of $O\left(10^{3}\right)$. This enables a new class of multiphase problems to be simulated, which were not previously possible. While we focus on one gas and two liquid components, the model can be extended to include more liquid components.

Our approach is based on the lattice Boltzmann method (LBM) [20,21], which has been shown to deliver reliable results, with quantitative agreement against experiments and other simulation methods, including on droplet dynamics [22-24], liquid phase separation [25,26], and flow through porous media [27]. In the LBM, interfacial forces can be implemented without explicit tracking of the interfaces, making it an elegant choice for studying mesoscopic interface dynamics in complex geometries.

Our key contribution over existing LBM models is a novel free energy functional that combines optimal equation of state for liquid-gas systems with double-well potentials to introduce multiple liquid components. The former, combined with the use of entropic lattice Boltzmann scheme [8], allows us to introduce significant density ratios, compared to other ternary free-energy LBM models $[17,18]$. The free energy formulation also ensures our model is thermodynamically consistent, unlike alternative approaches [14,28,29].

The capabilities of our new model are demonstrated using several static and dynamic problems. First, we find excellent agreement between the numerical and analytical liquid-gas coexistence curves as a function of temperature, proving the thermodynamic consistency of the model. Second, we illustrate how the liquid-liquid and liquidgas surface tensions can be flexibly tuned by simulating 
liquid lenses with varying Neumann angles. Finally, we simulate binary collisions between two immiscible droplets and show we capture many relevant features reported in experiments [30-34].

We introduce a free energy functional that consists of two parts, the bulk and interfacial contributions:

$$
\begin{gathered}
F=\int\left[f_{B}+f_{I}\right] d V, \\
f_{B}=\frac{\lambda_{1}}{2}\left(\Psi_{\mathrm{EOS}}(\rho)-\Psi_{0}\right)+ \\
\frac{\lambda_{2}}{2} C_{l 1}^{2}\left(1-C_{l 1}\right)^{2}+\frac{\lambda_{3}}{2} C_{l 2}^{2}\left(1-C_{l 2}\right)^{2}, \\
f_{I}=\frac{\kappa_{1}}{2}(\nabla \rho)^{2}+\frac{\kappa_{2}}{2}\left(\nabla C_{l 1}\right)^{2}+\frac{\kappa_{3}}{2}\left(\nabla C_{l 2}\right)^{2} .
\end{gathered}
$$

The bulk free energy density $f_{B}$ is designed to allow three distinct minima, corresponding to one gas and two liquid components, as illustrated in Fig. 1. $\Psi_{\mathrm{EOS}}(\rho)$ can be derived from integrating the liquid-gas equation of state (EOS), $p_{\mathrm{EOS}}=\rho\left(d \Psi_{\mathrm{EOS}} / d \rho\right)-\Psi_{\mathrm{EOS}}$, with coexisting liquidgas densities at $\rho_{l}$ and $\rho_{g}$. For concreteness, here we use Carnahan-Starling EOS, but our approach is flexible, and in the Supplemental Material [35] we describe the implementation of Peng-Robinson and van der Walls EOS. For Carnahan-Starling [36]:

$\Psi_{\mathrm{EOS}}=\rho\left(C-a \rho-\frac{8 R T(-6+b \rho)}{(-4+b \rho)^{2}}+R T \log (\rho)\right)$.

The constants $C$ and $\Psi_{0}$ are chosen such that $\Psi_{\mathrm{EOS}}\left(\rho_{g}\right)=$ $\Psi_{\mathrm{EOS}}\left(\rho_{l}\right)=\Psi_{0}$, ensuring common tangent construction is met between all coexisting fluid phases. We use $a=0.037$, $b=0.2$ and $R=1$. The critical temperature is $T_{c}=$ $0.3373(a / b R)$, and the temperature $T$ governs the liquid-gas density ratio.

The second and third terms in Eq. (2) have the form of double well potentials with $C_{l 1}$ and $C_{l 2}$ the relative concentrations of the two liquid components. Established

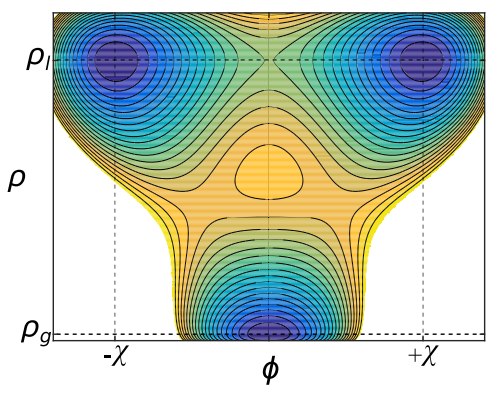

FIG. 1. Contour plot of the bulk free energy density $f_{B}$ as a function of two order parameters, $\rho$ and $\phi$. Three distinct minima exist, corresponding to a gas component at $\left(\rho_{g}, 0\right)$, and two liquid components at $\left(\rho_{l},+\chi\right)$ and $\left(\rho_{l},-\chi\right)$. works on critical phenomena show that such form is universal to describe the physics of continuous phase transitions close to the critical point [37], including for fluid mixtures. Away from the critical point, additional terms may be needed. However, so long as the details of the equation of state of the fluid mixtures is not important for the problem at hand, a large body of work in diffuse interface models for binary fluids has shown a double well potential is sufficient to capture interfacial dynamics with excellent agreement against both theory and experimental results, such as for droplet dynamics [22,23] and coarsening in phase separation $[25,26]$. This is the case for the examples studied here, and the double well potentials are therefore chosen as the simplest model possible.

Each double well term has two minima at $C_{\text {l\# }}=0$ (component absent) and $C_{l \#}=1$ (present). We also define the relative concentration of the gas phase as $C_{g}=$ $\left(\rho-\rho_{l}\right) /\left(\rho_{g}-\rho_{l}\right)$, which is 0 for $\rho=\rho_{l}$ and 1 for $\rho=\rho_{g}$. Given the constraint $C_{g}+C_{l 1}+C_{l 2}=1$, there are two independent order parameters: the density $\rho$ and the phase field $\phi$. The relative concentrations are related to the density and phase field via $C_{l 1}=\frac{1}{2}\left[1+\phi / \chi-\left(\rho-\rho_{l}\right) /\right.$ $\left.\left(\rho_{g}-\rho_{l}\right)\right]$ and $C_{l 2}=\frac{1}{2}\left[1-\phi / \chi-\left(\rho-\rho_{l}\right) /\left(\rho_{g}-\rho_{l}\right)\right]$, with $\chi$ a constant scaling parameter for $\phi$. Our free energy functional has three minima at $\left(\rho_{g}, 0\right),\left(\rho_{l},+\chi\right)$ and $\left(\rho_{l},-\chi\right)$.

For the interfacial free energy density, $f_{I}$, all three terms in Eq. (3) are necessary because there are three independent surface tensions in ternary systems. Upon expanding $C_{l \#}$ in terms of $\rho$ and $\phi$, we can rewrite $f_{I}$ as

$$
\begin{aligned}
f_{I}= & \left(\frac{\kappa_{1}}{2}+\frac{\kappa_{2}+\kappa_{3}}{8\left(\rho_{g}-\rho_{l}\right)^{2}}\right)(\boldsymbol{\nabla} \rho)^{2}+\frac{\kappa_{2}+\kappa_{3}}{8 \chi^{2}}(\boldsymbol{\nabla} \phi)^{2} \\
& +\frac{\kappa_{3}-\kappa_{2}}{4 \chi\left(\rho_{g}-\rho_{l}\right)}(\boldsymbol{\nabla} \rho \cdot \boldsymbol{\nabla} \phi) .
\end{aligned}
$$

We vary the $\lambda$ parameters in Eq. (2) and $\kappa$ parameters in Eq. (3) to tune the surface tensions and interfacial widths of the three fluid interfaces.

The continuum equations of motion for the fluid are the continuity, Navier-Stokes, and Cahn-Hilliard equations:

$$
\begin{gathered}
\partial_{t} \rho+\boldsymbol{\nabla} \cdot(\rho \boldsymbol{v})=0, \\
\partial_{t}(\rho \boldsymbol{v})+\boldsymbol{\nabla} \cdot(\rho \boldsymbol{v} \otimes \boldsymbol{v})=-\boldsymbol{\nabla} \cdot \boldsymbol{P}+\boldsymbol{\nabla} \cdot\left[\eta\left(\nabla \boldsymbol{v}+\nabla \boldsymbol{v}^{\boldsymbol{T}}\right)\right], \\
\partial_{t} \phi+\boldsymbol{\nabla} \cdot(\phi \boldsymbol{v})=M \nabla^{2} \mu_{\phi} .
\end{gathered}
$$

$v$ is the fluid velocity and $\eta$ is the dynamic viscosity that depends on the local density and phase field. For simplicity, we employ constant mobility parameter $M$, though in general it can depend on the local density and phase field [38]. The thermodynamics of ternary fluids, described by the free energy functional $F$ in Eq. (1), enter the equations 


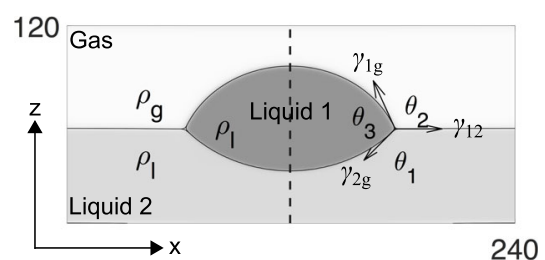

(a)

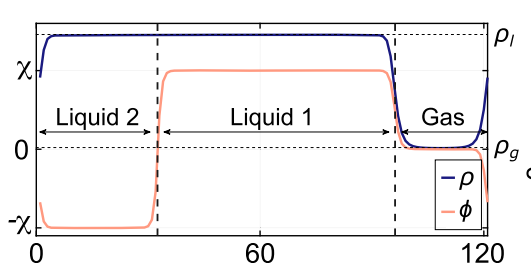

(b)

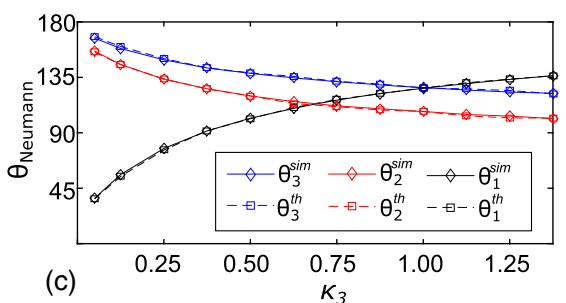

(c)

FIG. 2. (a) Simulation of a liquid 1 droplet suspended at the interface between liquid 2 and the gas phase. The Neumann angles $\theta_{1}, \theta_{2}$, $\theta_{3}$ are a consequence of mechanical equilibrium between the interfacial tensions. (b) The variation of the density $\rho$ and phase field $\phi$ across the vertical dashed line shown in (a). (c) Variation of the Neumann angles by varying $\kappa_{3}$. We fix $\lambda_{3}=3.125 \times \kappa_{3}, \lambda_{1}=0.6$, $\kappa_{1}=10^{-3}, \kappa_{2}=1.0, \lambda_{2}=3.125 \times \kappa_{2}, T_{r}=T / T_{c}=0.69$ and $\chi=5.0$.

of motion through the chemical potentials, $\mu_{\rho}=\delta F /\left.\delta \rho\right|_{T, \phi}$ and $\mu_{\phi}=\delta F /\left.\delta \phi\right|_{T, \rho}$, and the pressure tensor, $\boldsymbol{\nabla} \cdot \boldsymbol{P}=$ $\rho \boldsymbol{\nabla} \mu_{\rho}+\phi \boldsymbol{\nabla} \mu_{\phi}$. To solve the equations of motion, we introduce two sets of distribution functions in our LBM scheme, evolving the density and phase field. For the former, we employ the entropic lattice Boltzmann method, augmented with an exact-difference forcing term [8]. For the latter we use a standard BGK (Bhatnagar-Gross-Krook) scheme [21]. We provide the details of our LBM implementation in the Supplemental Material [35], including the expressions for the chemical potentials and pressure tensor.

To demonstrate the accuracy and broad range of surface tension ratios allowed in our model, we simulate a liquid lens, where a droplet of liquid 1 is suspended at the interface between liquid 2 and the gas phase, as shown in Fig. 2(a). We show the profiles of the density $\rho$ and phase field $\phi$ across the liquid lens configuration in Fig. 2(b). At the interface between liquids 1 and 2, $\rho$ remains constant at $\rho_{l}$, while $\phi$ transforms smoothly between $-\chi$ and $\chi$. Both $\rho$ and $\phi$ vary at the interface between any of the liquids and the gas.

At equilibrium, force balance between the surface tensions at the three phase contact line leads to a distinct set of angles known as the Neumann angles. Mathematically, $\gamma_{12} / \sin \left(\theta_{3}\right)=\gamma_{2 g} / \sin \left(\theta_{2}\right)=\gamma_{1 g} / \sin \left(\theta_{1}\right)$. To test this relation we vary the value of $\kappa_{3}$, while keeping $\lambda_{3}=3.125 \times \kappa_{3}$ and other simulation parameters (see caption of Fig. 2) constant. For all simulations shown here, we also set the kinematic viscosity $\nu=\eta / \rho=0.167$, and mobility parameter $M=0.5$. Figure 2(c) shows the Neumann angles calculated in two different ways. First, we measure the Neumann angles geometrically (diamond symbol) from our liquid lens simulations. Second, we use Laplace pressure tests to independently measure surface tensions for all permutations of the interfaces (see Supplemental Material [35]), and subsequently compute the expected Neumann angles (square symbol). The agreement is excellent, with typical deviations of $<3^{\circ}$. Similar agreement is observed upon varying other parameters.

In addition to partial wetting states, where the Neumann triangle is formed, our model allows simulations of full wetting states. To demonstrate this, in the Supplemental Material [35], we present simulation results of two droplets where $\gamma_{1 g}+\gamma_{12}<\gamma_{2 g}$. The droplets are initialized such that they are just touching each other. As dictated by thermodynamics, the simulation shows that the liquid 2 droplet becomes fully encapsulated by the liquid 1 droplet.

A wide range of density ratios can be simulated by tuning the temperature $T$ in the equation of state, Eq. (4). Figure 3 shows the coexistence curve for the Carnahan-Starling EOS. The left (right) branches correspond to the gas (liquid) densities. Good agreement is obtained between the analytical solution from Maxwell construction (line) and the numerical results (dots). The lowest temperature we can robustly simulate is $T=0.61 T_{c}$, corresponding to a numerical density ratio of $O\left(10^{3}\right)$. In the Supplemental Material [35] we also show that high density ratios can be achieved with Peng-Robinson and van der Walls EOS.

We now present simulation results of collisions between two immiscible droplets. In comparison to the more commonly studied problem of collisions between miscible droplets of the same materials (e.g., Refs. [8,9]), the collision outcomes for immiscible droplets are much richer. Here we show three regimes observed in experiments: bouncing, adhesive, and insertive collisions, and their transitions. To our best knowledge, this is the first time they have been simulated using the LBM. We will focus on generic features of the drop collisions. Systematic studies, including parameter matching against experiments, will be presented elsewhere.

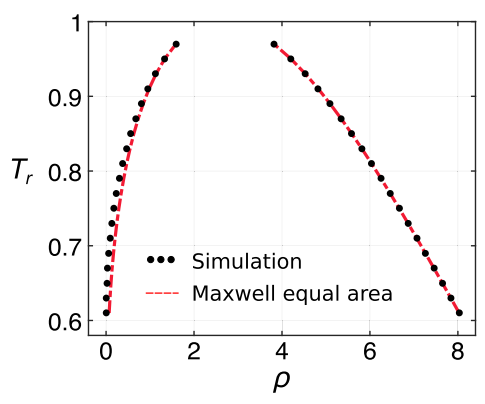

FIG. 3. The coexistence curve in the numerically stable regime for Carnahan-Starling EOS as a function of the reduced temperature $T_{r}=T / T_{c}$. 


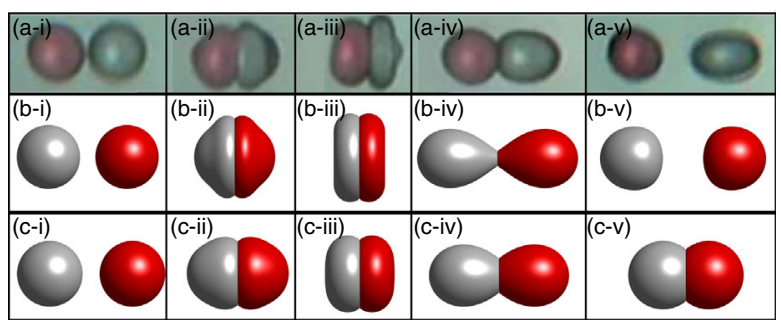

FIG. 4. (a) An experimental example of bouncing collision between two immiscible droplets (water and diesel oil) [31]. (b), (c) Transition from bouncing (b) to adhesive (c) collisions can be induced by decreasing the droplets' velocities.

We first consider bouncing collision. Figure 4(a) shows an experimental example where the two droplets are water and diesel oil [31]. As the droplets collide (columns ii and iii), we observe compression in the drop shapes parallel to the collision direction and radial expansions perpendicular to the collision direction. This is followed by retraction in the radial direction (column iv), and if there is sufficient kinetic energy, the two droplets bounce off and become separated (column v). Our simulations show this sequence is ubiquitous for head-on bouncing collisions. Figure 4(b) shows one such case at $\mathrm{We}_{1}=\mathrm{We}_{2}=20.8$ and $\mathrm{Re}_{1}=$ $\mathrm{Re}_{2}=72.0$, where $\mathrm{We}_{i}=\rho_{i} V_{r}^{2} D_{i} / \gamma_{i g}, \operatorname{Re}_{i}=\rho_{i} V_{r} D_{i} / \eta_{i}$, and $V_{r}$ is the relative droplet velocity. Here the two droplets have symmetric properties and we use $\gamma_{12} / \gamma_{2 g}=1.33$. We set $T=0.65 T_{c}$ for the rest of the Letter, corresponding to a density ratio of $\rho_{l} / \rho_{g} \simeq 150$. For the cases shown here, the results do not sensitively depend on the density ratio beyond $\rho_{l} / \rho_{g} \sim 100$. This is illustrated explicitly in the Supplemental Material [35] by comparing the results in Fig. 4 to those obtained using $T_{r}=0.61\left(\rho_{l} / \rho_{g} \simeq 1000\right)$.

By reducing the droplets' velocities, we observe a transition from bouncing to adhesive collision, shown in Fig. 4(c) for $\mathrm{We}_{1}=\mathrm{We}_{2}=5.6$ and $\mathrm{Re}_{1}=\mathrm{Re}_{2}=36.0$. Qualitatively the initial collision dynamics is similar between rows (b) and (c). However, at column (iv) there is not enough kinetic energy for the droplets to detach. Subsequently the compound droplet oscillates until it relaxes to its equilibrium configuration, determined by the Neumann triangle. Animations of the drop collisions in Figs. 4(b) and (c) are provided as Supplemental Material [35]. Adhesive collision between two immiscible droplets with similar liquid-gas surface tension has been observed experimentally for diesel and ethanol droplets [32].

A powerful advantage of our model is that it covers a wide range of surface tension ratios. We can now consider the asymmetric case where the liquid-gas surface tension of droplet 2 is considerably larger than that for droplet 1 , yet it does not correspond to the full wetting state. Figures 5(b)-(e) show the case where $\gamma_{12} / \gamma_{2 g}=0.54$ and $\gamma_{1 g} / \gamma_{2 g}=0.49$, with normalized spreading parameter $S=1-\left(\gamma_{1 g}+\gamma_{12}\right) / \gamma_{2 g}=-0.029$. In agreement with

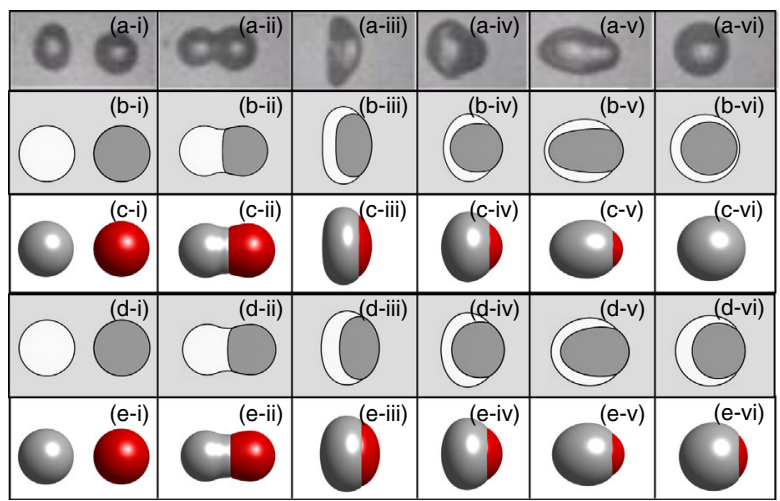

FIG. 5. (a) An experimental example of insertive collision between two immiscible droplets (water and n-hexadecane) [30], where one droplet is fully encapsulated by the other. Transition from insertive $[(b),(c)]$ to adhesive $[(d),(e)]$ collisions can be induced by decreasing the droplets' velocities. Rows (b) and (d) are cross sections of the drops in rows (c) and (e).

experimental observations [30], we observe a transition between adhesive and insertive collisions by varying the impact velocities.

An experimental example of insertive collision is shown in Fig. 5(a) for water and n-hexadecane [30]. For comparison, Figs. 5(b) and (c) show the typical dynamical sequence observed in our simulations, with $\mathrm{We}_{1}=16.4$, $\mathrm{We}_{2}=6.1$, and $\operatorname{Re}_{1}=\mathrm{Re}_{2}=45.0$. Upon collision, the composite droplet expands radially (column iii), followed by contraction in the radial direction (column iv) and elongation in the collision axis (column v). The oscillation between the prolate and oblate shapes can sustain several periods (videos in the Supplemental Material [35]), accompanied by the propagation of the three-phase contact line until the high surface tension droplet is fully encapsulated (column vi).

The transition from insertive to adhesive collision can be induced by decreasing the droplets' velocities. In Figs. 5(d) and (e), we present the case where $\mathrm{We}_{1}=4.4, \mathrm{We}_{2}=2.2$, and $\operatorname{Re}_{1}=\operatorname{Re}_{2}=27.0$. Initially the contact line propagates to cover the high surface tension droplet as the composite droplet oscillates between the prolate and oblate shapes (videos in the Supplemental Material [35]). Since the kinetic energy is insufficient to drive full encapsulation, the contact line eventually recedes and the droplet relaxes to its equilibrium shape (column vi). In the Supplemental Material [35] we further show the critical velocity for the transition between insertive and adhesive collisions increases as the normalized spreading parameter becomes more negative.

To conclude, we presented a strategy for modeling ternary multiphase multicomponent flows by combining a novel free energy formulation and the use of the entropic LBM scheme. Our approach allows significant density ratios, up to the order of $O\left(10^{3}\right)$, and a broad range of surface tension ratios, covering both partial and full wetting 
states, to be simulated. These flexibilities open up a number of applications, which were not previously possible. As an example, we demonstrated the bouncing, adhesive, and insertive regimes for binary collisions between immiscible droplets. Our method can meet the gap in systematic computational work for such collision dynamics, to complement the rich body of existing experimental studies [30-34]. Other applications are numerous, including drop impact on immiscible liquid film [2], advanced oil recovery [3], and liquid impregnated surfaces [4-7].

Here we have assumed the liquids to have the same density. This is justifiable in most water-oil-gas systems where the liquid-liquid density ratio is several orders of magnitude smaller than the liquid-gas density ratio. A useful future extension is to allow all density ratios to be varied independently. Our model can also be generalized to include more liquid components, by introducing additional double well potential and gradient terms in the bulk and interfacial free energy densities respectively. Another key avenue for future work is the interactions between ternary flows and complex solid surfaces. Our model is compatible with various approaches to introduce wetting boundary conditions [45-47].

We acknowledge funding from Procter \& Gamble (H. K.), EPSRC (H. K.; Grant No. EP/P007139/1) and SNF (I. K.; Grant No. 200021_172640).

The data sets generated and/or analyzed during the current study are available from $\mathrm{H}$. K. on reasonable request.

*karlin@lav.mavt.ethz.ch

†halim.kusumaatmaja@durham.ac.uk

[1] C. Wang, C. Lin, W. Hung, W. Huang, and C. Law, Combust. Sci. Technol. 176, 71 (2004).

[2] D. W. Murphy, C. Li, V. d'Albignac, D. Morra, and J. Katz, J. Fluid Mech. 780, 536 (2015).

[3] M. H. Holtz et al., in SPE Improved Oil Recovery Conference, Tulsa, Oklahoma, USA, 2016 (Society of Petroleum Engineers, Richardson, TX, 2016).

[4] A. Lafuma and D. Quere, Europhys. Lett. 96, 56001 (2011).

[5] J. D. Smith, R. Dhiman, S. Anand, E. Reza-Garduno, R. E. Cohen, G. H. McKinley, and K. K. Varanasi, Soft Matter 9 , 1772 (2013).

[6] T.-S. Wong, S. H. Kang, S. K. Y. Tang, E. J. Smythe, B. D. Hatton, A. Grinthal, and J. Aizenberg, Nature (London) 477, 443 (2011).

[7] C. Semprebon, G. McHale, and H. Kusumaatmaja, Soft Matter 13, 101 (2017).

[8] A. Mazloomi M, S. S. Chikatamarla, and I. V. Karlin, Phys. Rev. Lett. 114, 174502 (2015).

[9] D. Lycett-Brown, K. H. Luo, R. Liu, and P. Lv, Phys. Fluids 26, 023303 (2014).

[10] T. Inamuro, S. Tajima, and F. Ogino, Int. J. Heat Mass Transfer 47, 4649 (2004).

[11] T. Lee and C.-L. Lin, J. Comput. Phys. 206, 16 (2005).
[12] A. Fakhari, D. Bolster, and L.-S. Luo, J. Comput. Phys. 341, 22 (2017).

[13] A. K. Gunstensen, D. H. Rothman, S. Zaleski, and G. Zanetti, Phys. Rev. A 43, 4320 (1991).

[14] X. Shan and H. Chen, Phys. Rev. E 47, 1815 (1993).

[15] J. Blowey, M. I. M. Copetti, and C. Elliott, IMA J. Numer. Anal. 16, 111 (1996).

[16] F. Boyer and C. Lapuerta, ESAIM: Math. Model. Num. Anal. 40, 653 (2006).

[17] C. Semprebon, T. Krüger, and H. Kusumaatmaja, Phys. Rev. E 93, 033305 (2016).

[18] H. Liang, B. C. Shi, and Z. H. Chai, Phys. Rev. E 93, 013308 (2016).

[19] S. Dong, J. Comput. Phys. 338, 21 (2017).

[20] S. Succi, The Lattice Boltzmann Equation: For fluid Dynamics and Beyond (OUP, Oxford, 2001).

[21] T. Krüger, H. Kusumaatmaja, A. Kuzmin, O. Shardt, G. Silva, and E.M. Viggen, The Lattice Boltzmann Method: Principles and Practice (Springer, New York, 2016).

[22] Y. Liu, M. Andrew, J. Li, J. M. Yeomans, and Z. Wang, Nat. Commun. 6, 10034 (2015).

[23] T. Lee and L. Liu, J. Comput. Phys. 229, 8045 (2010).

[24] S. Varagnolo, D. Ferraro, P. Fantinel, M. Pierno, G. Mistura, G. Amati, L. Biferale, and M. Sbragaglia, Phys. Rev. Lett. 111, 066101 (2013).

[25] A. J. Wagner and J. M. Yeomans, Phys. Rev. Lett. 80, 1429 (1998).

[26] V. M. Kendon, J.-C. Desplat, P. Bladon, and M. E. Cates, Phys. Rev. Lett. 83, 576 (1999).

[27] H. Liu, Q. Kang, C. R. Leonardi, S. Schmieschek, A. Narváez, B. D. Jones, J. R. Williams, A. J. Valocchi, and J. Harting, Comput. Geosci. 20, 777 (2016).

[28] J. Bao and L. Schaefer, Appl. Math. Model. 37, 1860 (2013).

[29] Q. Li and K. H. Luo, Appl. Therm. Eng. 72, 56 (2014).

[30] C. H. Wang, C. Z. Lin, W. G. Hung, W. C. Huang, and C. K. Law, Combust. Sci. Technol. 176, 71 (2004).

[31] R.-H. Chen and C.-T. Chen, Exp. Fluids 41, 453 (2006).

[32] R.-H. Chen, Appl. Therm. Eng. 27, 604 (2007).

[33] I. V. Roisman, C. Planchette, E. Lorenceau, and G. Brenn, J. Fluid Mech. 690, 512 (2012).

[34] K.-L. Pan, Y.-H. Tseng, J.-C. Chen, K.-L. Huang, C.-H. Wang, and M.-C. Lai, J. Fluid Mech. 799, 603 (2016).

[35] See Supplemental Material at http://link.aps.org/ supplemental/10.1103/PhysRevLett.120.234501 for additional discussions on the derivation of the chemical potentials and pressure tensor, the implementation of the lattice Boltzmann method, the liquid-gas coexistence curves for the van der Walls and Peng-Robinson EOS, the Laplace pressure test, full wetting states for the Neumann triangle, the transition between insertive and adhesive collisions, and the comparison between simulation results at two different density ratios. We also provide videos of the drop collisions in Figs. 4 and 5 as Supplemental Material.

[36] P. Yuan and L. Schaefer, Phys. Fluids 18, 042101 (2006).

[37] J. Cardy, Scaling and Renormalization in Statistical Physics (Cambridge University Press, Cambridge, England, 1996). 
[38] There have been several works dedicated to understanding the choice of the mobility parameter $M$, including on coarsening dynamics during phase separation $[39,40]$, droplet coalescence and breakup [41,42], and contact line motion of a fluid interface at a solid boundary [43,44].

[39] S. Dai and Q. Du, J. Comput. Phys. 310, 85 (2016).

[40] J. Zhu, L.-Q. Chen, J. Shen, and V. Tikare, Phys. Rev. E 60, 3564 (1999).

[41] D. Jacqmin, J. Comput. Phys. 155, 96 (1999).

[42] P. M. Dupuy, M. Fernandino, H. A. Jakobsen, and H. F. Svendsen, Comput. Math. Appl. 59, 2246 (2010).
[43] H. Kusumaatmaja, E. J. Hemingway, and S. M. Fielding, J. Fluid Mech. 788, 209 (2016).

[44] P. Yue, C. Zhou, and J. J. Feng, J. Fluid Mech. 645, 279 (2010).

[45] H. Ding and P. D. M. Spelt, Phys. Rev. E 75, 046708 (2007).

[46] H. Huang, D. T. Thorne Jr., M. G. Schaap, and M. C. Sukop, Phys. Rev. E 76, 066701 (2007).

[47] J.-J. Huang, H. Huang, and X. Wang, Int. J. Numer. Methods Fluids 77, 123 (2015). 